\title{
Enfermagem no contexto da COVID-19: mapeamento sistemático
}

\author{
Nursing in the context of COVID-19: systematic mapping \\ La enfermería en el contexto de la COVID-19: mapeo sistemático
}

Recebido: 12/08/2021 | Revisado: 24/08/2021 | Aceito: 27/08/2021 | Publicado: 29/08/2021

\author{
Thamires Carraro Gatto \\ ORCID: https://orcid.org/0000-0002-4667-8111 \\ Universidade Federal de São Carlos, Brasil \\ E-mail: thamiresgatto@estudante.ufscar.br \\ Vinicius Paglione Carasek \\ ORCID: https://orcid.org/0000-0002-9255-0338 \\ Universidade Federal de São Carlos, Brasil \\ E-mail: vinicius.paglione@estudante.ufscar.br \\ Matheus Gabriel de Melo Sérgio \\ ORCID: https://orcid.org/0000-0002-5735-3398 \\ Universidade Federal de São Carlos, Brasil \\ E-mail: matheus.sergio@estudante.ufscar.br \\ Carolina Guerra da Mota e Silva \\ ORCID: https://orcid.org/0000-0001-9787-5636 \\ Universidade Federal de São Carlos, Brasil \\ E-mail: carolinagms@estudante.ufscar.br \\ Mellina Yamamura \\ ORCID: https://orcid.org/0000-0001-5228-8788 \\ Universidade Federal de São Carlos, Brasil \\ E-mail: mellina@ufscar.br
}

\begin{abstract}
Resumo
Introdução: O trabalho da enfermagem encontra-se na linha de frente para combate à COVID-19. Essa classe de trabalhadores é essencial para o funcionamento de todos os sistemas de saúde, pois, apesar de todas as dificuldades enfrentadas em relação à profissão e à pressão existente devido à crise epidemiológica vivenciada, ainda contribui para a ciência com sua produção científica. Objetivo: Identificar a produção científica da enfermagem na temática da COVID-19. Métodos: Trata-se de um estudo da literatura que visa mapear, de forma sistemática, a produção científica da enfermagem no contexto da COVID-19. Os mapeamentos sistemáticos consistem em uma ferramenta que possibilita uma visão ampliada da estrutura da base de evidências perante uma questão de pesquisa. Dessa maneira, a pergunta de pesquisa será: Qual a produção científica da enfermagem na temática da COVID-19? Será elaborada uma estratégia de busca dos estudos, por meio da determinação dos melhores descritores e/ou palavras-chaves que poderão relacionar-se com a temática. Considerar-se-ão bases de dados de referência mundial para maior amplitude de busca. As buscas serão realizadas em período determinado, no qual todas as publicações serão acessadas, sem quaisquer restrições quanto ao espaço temporal. Para a seleção dos estudos, serão realizadas a leitura criteriosa de título, resumo e palavras-chave e, posteriormente, a adequação aos critérios de inclusão e exclusão estabelecidos. As evidências encontradas serão apresentadas de maneira narrativa, por meio de tabelas, diagramas e mapa temático a ser elaborado no software Arcgis.
\end{abstract}

Palavras-chave: Enfermagem; COVID-19; Mapeamento sistemático; Produção científica.

\begin{abstract}
Introduction: The nursing work is in the front line in the fight against COVID-19. This class of workers is essential for the functioning of all health systems, since despite all the difficulties faced, related to the profession and the existing pressure due to the epidemiological crisis experienced, it still contributes to science with its scientific production. Objective: To identify in the scientific production of nursing on the theme of COVID-19. Methods: This is a study of the literature that aims to map in a systematic manner the scientific production of nursing in the context of COVID19. The systematic mappings consist of a tool that enables an enlarged view of the evidence base structure facing a research question. In this way, the research question will be: What is the scientific production of nursing on the theme of COVID-19? A search strategy for studies will be drawn up through the determination of the best descriptors and/or keywords that may be related to the theme. Worldwide reference databases will be considered for greater range of search. The searches will be carried out in a specified period, in which all publications will be accessed, without any restrictions on the timeline. For the selection of studies, a careful reading of the title, abstract and keywords and subsequently the adequacy to the inclusion and exclusion criteria established will be carried out. The evidence found will be presented in a narrative manner, through tables, diagram and thematic map to be elaborated in the software Arcgis.
\end{abstract}


Keywords: Nursing; COVID-19; Systematic mapping; Scientific production.

\section{Resumen}

Introducción: El trabajo de la enfermería se encuentra en la línea de frente para combatir el COVID-19. Esta clase de trabajadores es esencial para el funcionamiento de todos los sistemas de salud, a pesar de todas las dificultades enfrentadas en relación con la profesión y la presión existente debido a la crisis epidemiológica vivida, sigue contribuyendo la ciencia con su producción científica. Objetivo: Identificar la producción científica de la enfermería en la temática de COVID-19. Métodos: Se trata de un estudio de la literatura que pretende mapear, sistemáticamente la producción científica de la enfermería en el contexto del COVID-19. Los mapeos sistemáticos son una herramienta que permite una visión ampliada de la estructura de la base de pruebas sobre una pregunta de investigación. Así, la pregunta de investigación será: ¿Cuál es la producción científica de la enfermería en materia de COVID-19? Se elaborará una estrategia de búsqueda para los estudios, determinando los mejores descriptores y/o palabras clave que puedan relacionarse con el tema. Se considerarán bases de datos de referencia mundial para una búsqueda más amplia. Las búsquedas se realizarán en un periodo determinado, en el que se accederá a todas las publicaciones, sin restricciones en cuanto al espacio temporal. Para la selección de los estudios, se leerán cuidadosamente el título, el resumen y las palabras clave y se establecerá la adecuación a los criterios de inclusión y exclusión. Las pruebas encontradas se presentarán de forma narrativa, mediante tablas, diagramas y mapa temático que se preparará en el software Arcgis.

Palabras clave: Enfermería; COVID-19; Cartografía sistemática; Producción científica.

\section{Introdução}

O SARS-Cov-2 é o terceiro vírus pertencente à família coronavidae a causar uma pandemia no mundo, o primeiro foi o SARS-CoV-1, responsável por um surto de coronavírus na China em 2002, e MERS, no Oriente Médio em 2012, respectivamente (Ahn et al., 2020). Desde a identificação do SARS-Cov-2, a sociedade em todo o mundo está sofrendo com altas taxas de mortalidade ocorridas pela doença, além de uma infinidade de restrições e sobrecarga de demandas aos serviços de saúde (Galsash, et al., 2020). Em escala mundial, até o dia 3 de julho de 2021 foram registradas mais de 197.874 .812 pessoas infectadas pela COVID-19 e 4.217.400 óbitos. Dentre os países atingidos, os destaques em número de casos acumulados são os Estados Unidos, Índia e Brasil. Ao analisar os países que contabilizaram mais óbitos por COVID-19, o Brasil permanece em segundo lugar no ranking dos países com maior número de óbitos por COVID-19, pois até dia 11 de agosto de 2021, atingiu o número de 565.748 mortes e a somatória de 19.092 .832 pessoas infectadas. Frente aos dados alarmantes, é importante ressaltar a maior concentração de casos na região sudeste, com 38,34\% dos casos nacionais (Brasil, 2021).

Como ainda não há nenhum medicamento oficialmente aprovado que demonstre efeitos sobre o vírus, as únicas medidas para a contenção da pandemia são as de prevenção e vacinação (Carbone et al., 2021) e tais aspectos ultrapassam questões do sistema de saúde, pois envolve fatores sociais, econômicos, educacionais e, até mesmo políticos (Guner \& Hasanoglu \& Aktas, 2020).

Assim como em outras pandemias, a enfermagem está à frente e são insubstituíveis para o combate da COVID-19, pois centralizam muitas funções, conhecimentos e experiências (OMS, 2020). No Brasil, deve-se destacar o Sistema Único de Saúde (SUS), instituído pela Lei número 8.080/90, que é responsável por assistir 75\% da população brasileira e, junto a ele, temos o serviço da enfermagem que é essencial para a sustentação dessa estrutura (Neri da Silva \& Machado, 2019), uma vez que esses profissionais estão presentes na maioria das organizações do sistema de saúde (hospitais, UBS, UPAs e assim por diante), além de participarem da gestão e implementação de programas, como a Estratégia Saúde da Família, Serviço de Atendimento Médico de Urgência (SAMU), controle de imunização e doenças endêmicas, entre outros (Neri da Silva \& Machado, 2019).

Além de tal essencialidade para os serviços de saúde, a enfermagem, gradativamente, vem substituindo o conhecimento fundamentado na experiência adquirida pela prática baseada em evidências científicas, que consiste na 
investigação sistemática para o desenvolvimento de conhecimento sobre questões relacionadas com a profissão, que envolve experiência de cuidados ao longo da vida, promoção da saúde, cuidados preventivos ao fim da vida e coletivos envolvendo famílias e comunidades em diversos ambientes (Nkrumah et al., 2018).

Nesse sentindo, a prática da enfermagem está em foco há mais de um século, no entanto, tornou-se disciplina científica há pouco tempo (Nkrumah et al. 2018). Além disso, a qualificação profissional da área pode ser vislumbrada pela busca de novos conhecimentos científicos, visto que $80 \%$ dos enfermeiros fizeram algum curso de pós-graduação e 78,1\% dos técnicos e auxiliares pretendem cursar a graduação segundo pesquisa realizada recentemente (Neri da Silva \& Machado, 2019). Ademais, mesmo com todas as dificuldades e desvalias que essa profissão enfrenta, há um progresso substancial da enfermagem no quesito de produção de pesquisas, o que é de extrema importância para o enfrentamento de um momento pandêmico, como o atual (Frota et al., 2020).

Para tal, torna-se eminente que a capacidade resolutiva da Enfermagem ganhe destaque a cada dia e uma das formas de evidenciar essa potencialidade pode ser realizada pelo mapeamento de sua produção enquanto ciência e os efeitos extensamente positivos quando há profissionais de enfermagem com alto potencial crítico, científico tecnológico e que consiga abarcar as diversidades sociais de nosso planeta com equidade e universalidade. Assim, este protocolo apresenta como objetivo geral identificar na literatura nacional e internacional qual a produção científica da enfermagem na temática da COVID-19.

\section{Metodologia}

\section{Delineamento da Pesquisa}

Trata-se de um estudo da literatura que visa mapear de forma sistemática a produção científica da enfermagem no contexto da COVID-19. Os mapeamentos sistemáticos consistem em uma ferramenta que possibilita uma visão ampliada da estrutura da base de evidências perante a uma questão de pesquisa (Clapton \& Rutter \& Sharif, 2009; Grant \& Booth, 2009).

O procedimento de mapeamento sistemático possibilita organizar de forma sistematizada, clara e robusta, evidências sobre um determinado contexto, para auxiliar a tomada de decisão, bem como a possibilidade de levantamento de lacunas do conhecimento e potenciais questões que sejam passíveis de revisão. Ao contrário das revisões sistemáticas que se baseiam dos resultados para vincular relações quantitativas ou de metanálises, os mapeamentos sistemáticos permitem categorizar a literatura segundo os objetivos do estudo, o delineamento ou demais fatores que possuam significância no evento e questão (James \& Randall \& Haddaway, 2016)

Os mapeamentos sistemáticos auxiliam na tomada de decisões baseadas em evidências e têm ganhado destaque na área da saúde, apesar de ser comum em outras áreas. Diversos estudos foram realizados considerando esta perspectiva para auxiliar na identificação de evidências relevantes de lacunas de conhecimento e também no direcionamento de políticas e pesquisas futuras necessárias (Langoien et al., 2017; Kwasi et al., 2016; Filby \& Mcconville \& Portela, 2016).

Como o contexto da pandemia é ameaçador para todo o mundo e principalmente para a enfermagem, que está na linha de frente do enfrentamento contra a COVID-19, será realizado o mapeamento sistemático da literatura com o intuito de identificar diferentes procedimentos adotados pela categoria profissional, a fim de favorecer a prática clínica da enfermagem, o empoderamento da profissão enquanto ciência e também da produção do conhecimento científico oriundo desta situação que vem desafiando toda humanidade.

\section{Protocolo de Mapeamento Sistemático da Literatura}

De acordo com Clapton et al. (2009) e James, Randall e Haddaway (2016), o protocolo de mapeamento sistemático é realizado por etapas. Primeiramente, é necessário elaborar uma pergunta para nortear a pesquisa, a qual foi determinada a 
seguinte questão: Qual a produção científica da enfermagem na temática da COVID-19? Ainda, outras questões secundárias poderão ser estabelecidas para melhor caracterizar as publicações a serem obtidas.

Após a lapidação da pergunta de pesquisa, é preciso elaborar uma estratégia de busca dos estudos, por meio da determinação dos melhores descritores e/ou palavras-chaves que poderão relacionar-se com a temática. Depois, devem ser realizados testes para identificar se tais itens, realmente poderão representar a estratégia de busca para responder à questão de pesquisa. Operadores booleanos AND e OR devem ser utilizados e as bases de dados que melhor representarem os resultados da pergunta precisam ser definidas. Posteriormente foram realizadas análises em pares e, assim como demonstrado no Quadro 1, definiram-se estratégias de busca para cada base de acordo com os melhores resultados, a fim de responder à pergunta norteadora do estudo.

Quadro 1. Estratégias de busca de artigos utilizadas para a realização do mapeamento sistemático sobre a produção científica de enfermagem durante a pandemia da COVID-19.

\begin{tabular}{|c|c|}
\hline $\begin{array}{c}\text { Base de } \\
\text { dados }\end{array}$ & Streaming de Busca \\
\hline Scopus & $\begin{array}{l}\text { ALL ( "Nurses" OR "Nursing" OR "Nursing Staff" OR "Nursing Team" OR "Research, Nursing" ) AND ( } \\
\text { "COVID-19" OR "COVID19" OR "2019-nCoV infection" OR "coronavirus disease 2019" OR "COVID-19 } \\
\text { pandemic" OR "2019-nCoV disease" OR "2019 novel coronavirus disease" OR "2019 novel coronavirus } \\
\text { infection" OR "coronavirus disease-19" ) AND ( LIMIT-TO ( OA, "all" ) ) AND ( LIMIT-TO ( PUBYEAR, } \\
2020 \text { ) OR LIMIT-TO ( PUBYEAR , 2019) ) AND ( LIMIT-TO ( SUBJAREA, "NURS" ) ) }\end{array}$ \\
\hline $\begin{array}{l}\text { Web of } \\
\text { Science }\end{array}$ & 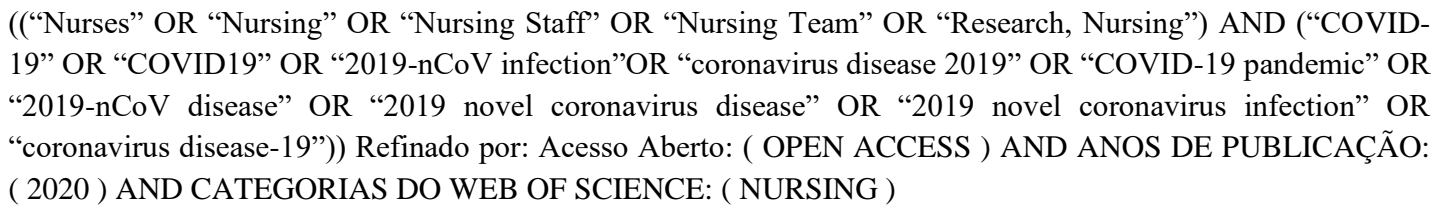 \\
\hline PubMed & 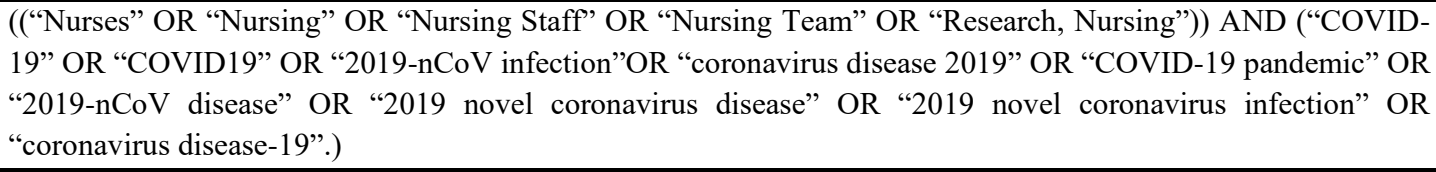 \\
\hline LILACS & $\begin{array}{l}\text { Nurses OR Nursing OR Nursing Staff OR Nursing Team OR Research, Nursing AND COVID-19 OR COVID19 } \\
\text { OR 2019-nCoV infection OR coronavirus disease } 2019 \text { OR COVID-19 pandemic OR 2019-nCoV disease OR } \\
2019 \text { novel coronavirus disease OR } 2019 \text { novel coronavirus infection OR coronavirus disease-19 }\end{array}$ \\
\hline CINAHL & $\begin{array}{l}\text { ("Nurses" OR "Nursing" OR "Nursing Staff” OR "Nursing Team" OR "Research, Nursing") AND ( "COVID- } \\
\text { 19" OR "COVID19" OR "2019-nCoV infection"OR "coronavirus disease 2019" OR "COVID-19 pandemic" OR } \\
\text { "2019-nCoV disease" OR "2019 novel coronavirus disease" OR "2019 novel coronavirus infection" OR } \\
\text { "coronavirus disease-19") }\end{array}$ \\
\hline
\end{tabular}

Fonte: Construção dos autores com base na estratégia de busca das bases de dados consultadas Scopus, Web o Science, PubMed, LILACS e CINHAL.

Após a realização das buscas serão inclusas as pesquisas encontradas nos idiomas inglês, espanhol e português, com abordagem quantitativa, qualitativa, métodos mistos, estudos primários, revisões sistemáticas, metanálises e/ou metassínteses e editoriais publicados em fontes indexadas que respondam à pergunta estabelecida. Não serão incluídas: obras em idiomas diferentes dos estabelecidos; literatura cinzenta, retrações, websites e propagandas veiculadas em mídias por não se tratarem de publicações com rigor científico. 
As buscas serão realizadas em período determinado devido a data de surgimento da COVID-19, no qual todas as publicações serão acessadas, sem quaisquer restrições quanto ao espaço temporal. Para a seleção dos estudos, será realizada a leitura criteriosa do título, resumo e palavras-chave e para definir a seleção final, buscar-se-á a leitura do artigo na íntegra.

Por se tratar de um mapeamento sistemático, serão consideradas variáveis básicas, como desenho do estudo, país de origem da pesquisa e profissionais e/ou população envolvida. As categorias de discussão serão agrupadas de acordo com os resultados encontrados nos estudos.

As evidências identificadas serão apresentadas de maneira narrativa, por meio de tabelas e diagrama (Tricco, 2018) e também através da visualização gráfica dos estudos em um mapa temático a ser elaborado no software Arcgis.

\section{Financiamento}

O presente trabalho foi realizado com apoio da Coordenação de Aperfeiçoamento de Pessoal de Nível Superior Brasil (CAPES) - Código de Financiamento 001.

\section{Referências}

Ahn, D. G., Shin, H. J., Kim, M. H., Lee, S., Kim, H. S., Myoung, J., \& Kim, S. J. (2020). Current status of epidemiology, diagnosis, therapeutics, and vaccines for novel coronavirus disease 2019 (COVID-19). Journal of Microbiology and Biotechnology 30(3):313-324. 10.4014/jmb.2003.03011.

Brasil. Ministério da Saúde. Secretaria de Vigilância em Saúde. (2021). Painel Coronavírus. https://covid.saude.gov.br/.

Brasil. Ministério da Saúde. Secretaria de Vigilância em Saúde. (2021). Centro de Operações de Emergência em Saúde Pública-COVID-19. Bol Epidemiol Especial, 70, 1-98, 2021a. https://www.gov.br/saude/pt-br/media/pdf/2021/julho/09/boletim_epidemiologico_covid_70-1.pdf.

Carbone, M., Lednicky, J., Xiao, S. Y., Venditti, M., \& Bucci, E. (2021). Coronavirus 2019 infectious disease epidemic: where we are, what can be done and hope for. Journal of Thoracic Oncology, 16(4), 546-571. 10.1016/j.jtho.2020.12.014.

Clapton, J., Rutter, D., \& Sharif, N. (2009). SCIE Systematic mapping guidance. SCIE.

Cofen (Conselho Federal de Enfermagem) (2021) Observatório da Enfermagem: profissionais infectados com covid-19 informado pelos enfermeiros responsáveis técnicos/coordenadores. http://observatoriodaenfermagem.cofen.gov.br/.

Filby, A. McConville. F. and Portela, A., (2016.) What prevents quality midwifery care? A systematic mapping of barriers in low and middle income countries from the provider perspective. PLoS One, 11(5), e0153391. 10.1371/ journal.pone.0153391.

Frota, M. A., Wermelinger, M. C. D. M. W., Vieira, L. J. E. D. S., Ximenes Neto, F. R. G., Queiroz, R. S. M., \& Amorim, R. F. D. (2019). Mapeando a formação do enfermeiro no Brasil: desafios para atuação em cenários complexos e globalizados. Ciência \& Saúde Coletiva, 25, 25-35. https://doi.org/10.1590/1413-81232020251.27672019.

Gallasch, C. H., da Cunha, M. L., de Souza Pereira, L. A., \& Silva-Junior, J. S. (2020). Prevenção relacionada à exposição ocupacional do profissional de saúde no cenário de COVID-19 [Prevention related to the occupational exposure of health professionals workers in the COVID-19 scenario][Prevención relacionada cone la exposición ocupacional de profesionales de la salud en el escenario COVID-19]. Revista Enfermagem UERJ, 28, 49596. https://doi.org/10.12957/reuerj.2020.49596.

Grant, M. J., \& Booth, A. (2009). A typology of reviews: an analysis of 14 review types and associated methodologies. Health information \& libraries journal, 26(2), 91-108. 10.1111/j.1471-1842.2009.00848.x.

Güner, H. R., Hasanoğlu, İ., \& Aktaş, F. (2020). COVID-19: Prevention and control measures in community. Turkish Journal of medical sciences, 50(SI-1), 571-577. 10.3906/sag-2004-146

James, K. L., Randall, N. P., \& Haddaway, N. R. (2016). A methodology for systematic mapping in environmental sciences Environ. Environmental evidence, v. 5, n. 1, p. 7. https://doi.org/10.1186/s13750-016-0059-6.

Langøien, L. J., Terragni, L., Rugseth, G., Nicolaou, M., Holdsworth, M., Stronks, K., \& Roos, G. (2017). Systematic mapping review of the factors influencing physical activity and sedentary behaviour in ethnic minority groups in Europe: a DEDIPAC study. International Journal of Behavioral Nutrition and Physical Activity, 14(1), 1-24. 10.1186/s12966-017-0554-3.

Nadeem, M. S., Zamzami, M. A., Choudhry, H., Murtaza, B. N., Kazmi, I., Ahmad, H., \& Shakoori, A. R. (2020). Origin, potential therapeutic targets and treatment for coronavirus disease (COVID-19). Pathogens, 9(4), 307. https://doi.org/10.3390/pathogens9040307.

Nkrumah, I., Atuhaire, C., Priebe, G., \& Cumber, S. N. (2018). Barriers for nurses' participation in and utilisation of clinical research in three hospitals within the Kumasi Metropolis, Ghana. The Pan African Medical Journal, 30. 10.11604/pamj.2018.30.24.15230. 
Research, Society and Development, v. 10, n. 11, e240101119378, 2021

(CC BY 4.0) | ISSN 2525-3409 | DOI: http://dx.doi.org/10.33448/rsd-v10i11.19378

Osei-Kwasi, H. A., Nicolaou, M., Powell, K., Terragni, L., Maes, L., Stronks, K., \& Holdsworth, M. (2016). Systematic mapping review of the factors influencing dietary behaviour in ethnic minority groups living in Europe: a DEDIPAC study. International Journal of Behavioral Nutrition and Physical Activity, 13(1), 1-17. https://doi.org/10.1186/s12966-016-0412-8.

Ren, S. Y., Wang, W. B., Hao, Y. G., Zhang, H. R., Wang, Z. C., Chen, Y. L., \& Gao, R. D. (2020). Stability and infectivity of coronaviruses in inanimate environments. World journal of clinical cases, 8(8), 1391-1399. 10.12998/wjcc.v8.i8.1391.

Setti, L., Passarini, F., De Gennaro, G., Barbieri, P., \& Perrone, M. G., M Borelli, Palmisani J, Gilio A, Piscitelli P, Alessandro Miani A. (2020) Airborne Transmission Route of COVID-19: Why 2 Meters/6 Feet of Inter-Personal Distance Could Not Be Enough. Int J Environ Res Public Health. 17(8), 2932. 10.3390/ijerph17082932.

Silva, M. C. N. D., \& Machado, M. H. (2019). Sistema de Saúde e Trabalho: desafios para a Enfermagem no Brasil. Ciência \& Saúde Coletiva, 25, 07-13. https://doi.org/10.1590/1413-81232020251.27572019.

Singhal, T. (2020). Uma revisão da doença de Coronavírus-2019 (COVID-19). Indian J Pediatr, 87, (4), 281-286. 10.1007/s12098-020-03263-6.

Soldati, G., Giannasi, G., Smargiassi, A., Inchingolo, R., \& Demi, L. (2020). Contrast-enhanced ultrasound in patients with COVID-19: pneumonia, acute respiratory distress syndrome, or something else? Journal of Ultrasound in Medicine, 39(12), 2483-2489. 10.1002/jum.15338.

Tricco, A. C., Lillie, E., Zarin, W., O'Brien, K. K., Colquhoun, H., Levac, D., \& Straus, S. E. (2018). PRISMA extension for scoping reviews (PRISMA-ScR): checklist and explanation. Annals of internal medicine, 169(7), 467-473. 10.7326/M18-0850.

Wong, S. H., Lui, R. N., \& Sung, J. J. (2020). Covid-19 and the digestive system. Journal of gastroenterology and hepatology, 35(5), 744-748. 10.1111/jgh.15047.

World Health Organization. (2020) World's Nursing 2020. https://www.who.int/publications-detail/nursing-report-2020. 